\title{
MODELO MATEMÁTICO APLICADO À IRRIGAÇÃO LOCALIZADA COM MICROTUBOS SOB REGIME DE ESCOAMENTO TURBULENTO ${ }^{1}$
}

\author{
WANDERLEY DE J. SOUZA ${ }^{2}$, TARLEI A. BOTREL ${ }^{3}$, \\ ALEXSANDRO C. DOS S. ALMEIDA ${ }^{4}$, CRISTINA B. CORREA ${ }^{5}$
}

\begin{abstract}
RESUMO: O presente trabalho teve como objetivos, desenvolver dois modelos matemáticos para dimensionamento de microtubos em irrigação localizada, operando sob regime de escoamento turbulento, nos quais foram incluídas as perdas de carga no microtubo, perda de carga localizada e perdas por energia de velocidade na saída do microtubo. Para a determinação da perda de carga localizada, obteve-se, por meio de dados de laboratório, valores de coeficientes $\mathrm{K}$ de dissipação de energia na entrada do microtubo. Os modelos foram avaliados com o dimensionamento de uma linha lateral de irrigação e tiveram ótimos desempenhos, tendo em vista que os dados coletados proporcionaram vazão média próxima da vazão de projeto, com coeficiente de determinação acima de $98 \%$, excelente uniformidade de distribuição, baixo erro médio absoluto e coeficiente de variação de vazão. Dessa forma, pode-se dizer que o sistema de irrigação com microtubos, em regime de escoamento turbulento, mostrou-se tecnicamente viável para utilização, e que os modelos matemáticos desenvolvidos foram adequados para a avaliação das perdas no sistema.
\end{abstract}

PALAVRAS-CHAVE: linha lateral, perda de carga, hidráulica.

\section{MATHEMATIC MODEL APPLIED TO DRIP IRRIGATION WITH MICROTUBS UNDER TURBULENT FLOW}

\begin{abstract}
The present work was carried out in order to develop two mathematics models for design drip irrigation with microtubs, under turbulent flow. In the models were taken account the local head loss in the microtub, local head loss and head loss due to velocity energy in the microtub outside. To determine the local head loss it was calculated the $\mathrm{K}$ coefficients of the energy dissipation at the entrance of the microtub through laboratory tests. The models were evaluated through the design of an irrigation lateral line, ant it was obtained great performance with determination coefficient of $98 \%$, the discharge observed were close to the projected discharge, excellent distribution uniformity, low mean absolute error and variation of discharge coefficient. According to the results, it can be conclude that the microtub drip irrigation operating under turbulent regime is technically feasible and the developed of mathematical models are adequate to evaluate the head loss in the system.
\end{abstract}

KEYWORDS: lateral line, head loss, hydraulic.

\section{INTRODUÇÃO}

O crescimento da população mundial e da atividade humana tem contribuído para o aumento do consumo de água e alimentos (CARARO \& BOTREL, 2007). Isso contribui para aumento na implantação de sistemas de irrigação. Dados apresentados por CHRISTOFIDIS (2007) mostram que, em 1980, a área irrigada no Brasil era de aproximadamente 1,60 milhão de hectares, sendo que,

\footnotetext{
${ }^{1}$ Parte da dissertação de Mestrado em Irrigação e Drenagem do primeiro autor.

${ }^{2}$ Eng $^{\mathrm{o}}$ Agrícola, Doutorando em Irrigação e Drenagem, Departamento de Engenharia de Biossistemas, ESALQ/USP, Piracicaba - SP, wjsouza@esalq.usp.br

${ }^{3}$ Eng $^{\mathrm{o}}$ Agrí́cola, Prof.Associado, Departamento de Engenharia de Biossistemas, ESALQ/USP, Piracicaba - SP.

${ }^{4}$ Eng ${ }^{0}$ Agrônomo, Doutorando em Irrigação e Drenagem, Departamento de Engenharia de Biossistemas, ESALQ/USP, Piracicaba SP.

${ }^{5}$ Eng $^{\mathrm{o}}$ de Alimentos, Mestranda em Ciência de Alimentos, Departamento de Agroindústria, ESALQ/USP, Piracicaba - SP.

Recebido pelo Conselho Editorial em: 14-11-2008
}

Aprovado pelo Conselho Editorial em: 27-1-2011 
para 2020, a previsão é de 5,65 milhões de hectares irrigados. Portanto, é aconselhável que se dê preferência por sistemas pressurizados de irrigação que utilizem a água de uma forma mais eficiente possível, como a irrigação localizada, sem comprometer a produção final (SOUZA et al., 2005) . Esta é, dentre as técnicas de irrigação, a que mais cresceu nos últimos anos, em função da maneira racional do uso da água (AIROLDI, 2003), portanto os microtubos podem ser uma alternativa viável para tal método, buscando baixo custo e alto nível tecnológico.

Apesar de o microtubo ter sido precursor na irrigação como gotejador de longo percurso, o Brasil ainda carece de estudos, sendo a literatura carente de informações a seu respeito. Para sua utilização, existem alguns fatores relevantes que devem ser considerados: fração da energia cinética (coeficiente K) devido à passagem do líquido de uma zona de maior área transversal para dentro do microtubo, que resultará na dissipação pontual de energia (Hf Localizado), dissipação de energia devido à velocidade de saída do líquido (Ev) e à passagem dentro do microtubo (Hf Tubo), uma vez que os outros parâmetros utilizados na equação que descreve a dissipação de energia são conhecidos. A determinação destes fatores pode ser feita em laboratório, com auxílio de equações básicas já consagradas na hidráulica, podendo ser utilizada a equação universal [eq.(1)], utilizando para regime turbulento o valor de $f$ apresentado na eq.(2), perda de carga localizada eq.(3) e energia de velocidade eq.(4), e avaliados em campo.

$$
\begin{aligned}
& \text { HfTubo }=\frac{8 f Q^{2} L}{\pi^{2} g D^{5}} \\
& f=0,31 \operatorname{Re}^{-0,25} \\
& H f \text { Localizado }=K \frac{16 Q^{2}}{2 \pi^{2} g D^{4}} \\
& E_{V}=\frac{16 Q^{2}}{2 \pi^{2} g D^{4}}
\end{aligned}
$$

em que:

Hf - energia dissipada ou "gasta", mca;

F - coeficiente de atrito em função do regime de escoamento, adimensional;

D - diâmetro interno da tubulação, m;

L - comprimento da tubulação, m;

$\mathrm{Q}$ - vazão, $\mathrm{m}^{3} \mathrm{~s}^{-1}$;

$\mathrm{g}$ - aceleração da gravidade, $\mathrm{m} \mathrm{s}^{-2}$;

$\mathrm{K}$ - coeficiente de perda de carga localizada, adimensional, e

Re - número de Reynolds, adimensional.

As características hidráulicas dos emissores em irrigação localizada constituem-se na relação vazão versus pressão na entrada, na uniformidade de fabricação, na uniformidade de distribuição da água (NASCIMENTO, 1999), ocorrendo menor variação da carga hidráulica na linha de derivação em declive, para maiores valores de uniformidade de emissão (SAAD \& MARCUSSI, 2006), e devem ser determinadas experimentalmente. Portanto, é preferível que se utilize um modelo matemático que correlacione vazão, pressão na entrada do microtubo, comprimento e diâmetro.

Nas últimas décadas, o uso de modelos de simulação tem sido uma opção interessante na pesquisa agrícola (ZANETTI et al., 2005), uma vez que, com as tecnologias existentes atualmente, métodos computacionais personalizados, é possível a introdução de modelos matemáticos que representem as energias dissipadas no processo de condução de um fluido em microtubo, para um dimensionamento que apresente rapidez e precisão nos cálculos. VERMEIREN \& JOBLING (1980) 
afirmam que a relação entre vazão, comprimento, energia piezométrica e diâmetro interno do microtubo pode ser representada em uma só equação.

Diante do exposto, pretendeu-se, com esta pesquisa, desenvolver um método alternativo de irrigação com microtubos operando em regime turbulento, além de se ter como objetivos desenvolver duas equações que representam modelos para dimensionamento de microtubos, nas quais estivessem explícitas a perda de carga no microtubo, perdas por energia de velocidade e localizada, e realizar a avaliação hidráulica das mesmas, em campo.

\section{MATERIAL E MÉTODOS}

Para este trabalho, utilizou - se de microtubos com diâmetro interno comercial de 1,00 mm (V), 0,80mm (A), 0,70 mm (L) e 0,60 mm (Az), e diâmetro externo de 2,50 mm. A determinação precisa do diâmetro interno do microtubo foi realizada por duas metodologias: utilizando um projetor de perfil com precisão de $1,00 \mu \mathrm{m}$, pertencente ao Departamento de Engenharia Rural da ESALQ/USP e por meio de medições hidráulicas.

Para medir o diâmetro pelo projetor de perfil, tomou-se uma amostra de cada microtubo e, com auxílio de um bisturi, cortaram-se transversalmente 60 amostras simples contendo comprimentos de aproximadamente $2,00 \mathrm{~mm}$. As partes cortadas foram colocadas no projetor de perfil, e medido o diâmetro interno para cada amostra simples.

Pelo método hidráulico, o diâmetro foi determinado utilizando-se de diferentes pressões de operação e comprimentos de microtubos. Os microtubos foram inseridos em um tubo de polietileno com diâmetro de $20 \mathrm{~mm}$ em nível. Para cada comprimento, o emissor funcionou com duas pressões de serviço diferentes, medidas com um manômetro de Bourdon. Em cada condição de pressão e comprimento, a vazão foi mensurada três vezes, sendo que, para cada valor coletado, mediu-se a temperatura da água.

A vazão dos emissores foi determinada com base no volume de água coletada durante 30 minutos. Os valores de pressão e comprimentos com os quais se realizaram os testes e a determinação do diâmetro interno estão na Tabela 1. Na Figura 1, ilustram-se os detalhes da linha lateral com o microtubo inserido, desde a passagem da água da linha lateral para o microtubo (ponto 1) até a saída (ponto 3), para a determinação do coeficiente $K$ de dissipação de energia localizada.

TABELA 1. Pressões e comprimentos em que foram realizados os testes hidráulicos para determinação do diâmetro interno dos microtubos. Pressures and lengths in where the hydraulics tests were made to determine the inside diameter of the microtub.

\begin{tabular}{ccccc}
\hline \multirow{2}{*}{ Pressão $(\mathrm{kPa})$} & \multicolumn{4}{c}{ Comprimentos dos Microtubos $(\mathrm{m})$} \\
\cline { 2 - 5 } & $\mathrm{V}$ & $\mathrm{A}$ & $\mathrm{L}$ & $\mathrm{Az}$ \\
\hline 17,61 & 4,50 & 5,00 & 6,00 & 10,00 \\
110,67 & 4,00 & 5,00 & 7,00 & 10,00 \\
120,18 & 4,00 & 5,00 & 7,00 & 10,00 \\
155,80 & 4,00 & 5,00 & 7,00 & 10,00 \\
202,87 & 5,00 & 6,00 & 8,00 & 11,00 \\
249,40 & 5,00 & 6,00 & 8,00 & 11,00 \\
297,43 & 6,00 & 7,00 & 9,00 & 12,00 \\
345,65 & 6,00 & 7,00 & 9,00 & 12,00 \\
\hline
\end{tabular}




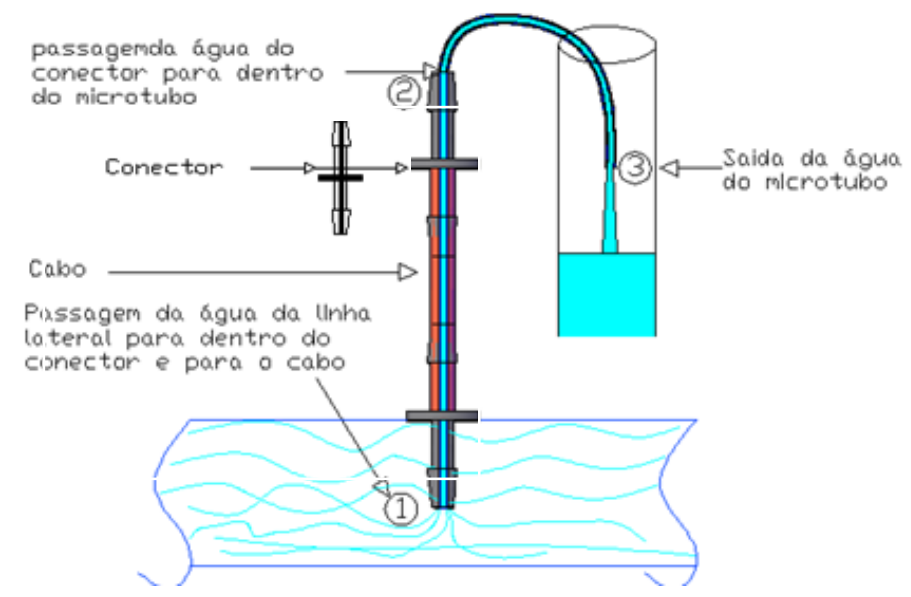

FIGURA 1. Representação esquemática do caminho percorrido pela água desde a entrada no conector até a saída do microtubo, para a determinação do coeficiente $\mathrm{K}$ de perda de carga localizada. Schematic representation of the water path from the entrance of the connector to the exit of the microtub, to determine the $K$ coefficient of the localized head loss.

Os microtubos foram inseridos em um conector de 2,4 $\mathrm{mm}$ de diâmetro interno, que também foi inserido em uma linha lateral de polietileno com diâmetro de $20 \mathrm{~mm}$, por intermédio de um cabo de 3,80 $\mathrm{mm}$ de diâmetro interno (Figura 1), em furos realizados com um furador de 2,00 $\mathrm{mm}$ de diâmetro externo. Para a determinação dos coeficientes K, representados pela eq.(13) a seguir, os microtubos foram seccionados em comprimentos de 0,$10 ; 0,15 ; 0,20 ; 0,25$ e $0,30 \mathrm{~m}$.

Pela Figura 1, pode-se compreender melhor a eq. (12) demonstrada a partir da eq.(5):

$$
P_{1}=H f C a b o+H f T u b o+{\frac{V_{3}}{2 g}}^{2}+\text { HfLocalizada }
$$

em que,

$\mathrm{P}_{1}$ - carga de pressão na entrada do cabo, no ponto 1 ;

$\mathrm{V}_{3}$ - velocidade da água na saída do microtubo;

$\mathrm{g}$ - aceleração da gravidade;

H f Cabo - dissipação de energia no cabo;

H f Tubo - dissipação de energia no microtubo, e

H f Localizada - dissipação de energia localizada.

As equações utilizadas foram a de Darci-Weistbach para o cálculo de HfCaba e HfTubo, e as equações de dissipação de energia em função da velocidade na saída do microtubo $\left(E_{V}\right)$ e localizada, dadas por:

$$
\begin{aligned}
& H f C a b o=f \frac{L_{1-2}}{D_{1}} \frac{V_{2}{ }^{2}}{2 g} \\
& H f T u b o=f \frac{L_{2-3}}{D_{2}}{\frac{V_{3}}{2 g}}^{2} \\
& E_{V}={\frac{V_{3}}{2 g}}^{2}
\end{aligned}
$$




$$
\begin{aligned}
& H f \text { Localizada }=K \frac{V_{3}^{2}}{2 g} \\
& f=\frac{0,316 v^{0,25}}{(V D)^{0,25}} \\
& V=\frac{4 Q}{\pi D^{2}}
\end{aligned}
$$

Fazendo-se H como sendo a carga de pressão inicial na linha lateral, substituindo (11) em (6), (7), (8), (9), (10), e isolando - se o coeficiente K de dissipação, tem-se:

$$
K=\left\{H-\left[\begin{array}{c}
\left.\left[\frac{0,316}{2 g}\left(\frac{4}{\pi}\right)^{1,75} \frac{v^{0,25}}{1}\left(\frac{Q^{1,75} L_{1-2}}{D_{1}^{4,75}}\right)\right]+\right] \\
{\left[\frac{0,316}{2 g}\left(\frac{4}{\pi}\right)^{1,75} \frac{v^{0,25}}{1}\left(\frac{Q^{1,75} L_{2-3}}{\left.D_{2}^{4,75}\right)}\right]+\right.} \\
{\left[\frac{16}{2 \pi^{2} g}\left(\frac{Q^{2}}{D_{2}{ }^{4}}\right)\right]}
\end{array}\right\} *\left[\frac{2 \pi^{2} g}{16}\left(\frac{D_{2}^{4}}{Q^{2}}\right)\right]\right.
$$

em que,

$\mathrm{K}$ - constante de dissipação de energia na entrada do microtubo, adimensional;

$\mathrm{H}$ - carga de pressão na entrada no ponto 1, m.c.a;

$\mathrm{g}$ - aceleração da gravidade $\mathrm{m} \mathrm{s}^{-2}$;

$\mathrm{Q}$ - vazão a saída do microtubo, $\mathrm{m}^{3} \mathrm{~s}^{-1}$;

$\mathrm{v}$ - viscosidade cinemática, $\mathrm{m}^{2} \mathrm{~s}^{-1}$;

$\mathrm{D}_{1}$ - diâmetro do cabo, m;

$\mathrm{D}_{2}$ - diâmetro do microtubo, $\mathrm{m}$;

$\mathrm{L}_{1-2}$ - comprimento do cabo, $\mathrm{m}$, e

$\mathrm{L}_{2-3}$ - comprimento do microtubo, $\mathrm{m}$.

Para cada comprimento de microtubo inserido na linha lateral, utilizou-se de dezesseis diferentes pressões, com as quais o volume de água foi coletado em um tempo não inferior a três minutos e pesado. Cada coleta se repetiu três vezes; dessa forma, o total de dados coletados para cada microtubo em todos os comprimentos foi de 240 . Para cada vazão coletada, monitorou-se a temperatura da água para a consideração da viscosidade cinemática na equação de determinação do valor de K [eq.(12)]. Em seguida, por intermédio de uma equação ajustada em uma planilha eletrônica, foram obtidos os valores de $\mathrm{K}$ em função do número de Reynolds para cada microtubo em estudo.

Operando em regime turbulento, determinaram-se dois modelos Matemáticos - hidráulicos para a estimativa dos comprimentos dos microtubos. Um modelo denominado UNIVERSAL, eq.(16), proposto a partir da equação de Darci-Weistbach em função das energias dissipadas no cabo e no microtubo, e das equações de dissipação de energia localizada e em função da velocidade da água na saída do microtubo (Ev). Outro, denominado WSBOTREL, eq.(20), utilizando as mesmas equações, porém adaptadas aos dados obtidos em laboratório. A Figura 2 auxilia no entendimento das eqs.(18) e (26), obtidas a partir de (13). 


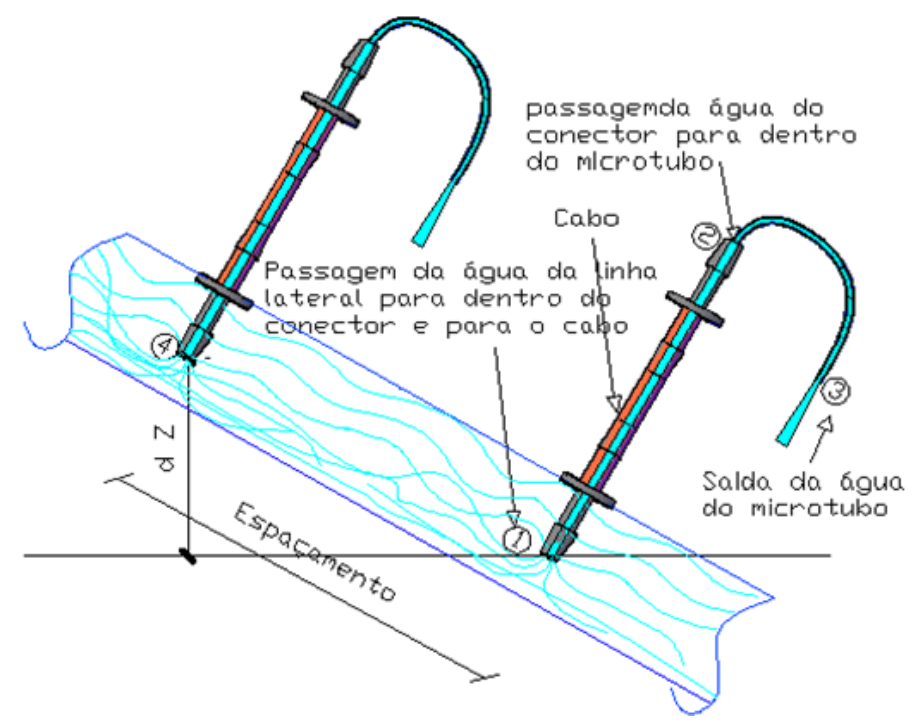

FIGURA 2. Ilustração do escoamento no sistema com microtubos, do ponto 4 (microtubo 1) até a saída no ponto 3 (microtubo 2). Illustration of flow in the system with microtubs, from point 4 (microtub 1) to the exit in point 3 ( microtub 2).

\section{Modelo 1 (UNIVERSAL)}

Equação de Bernoulli aplicada entre os pontos 1 e 3 da Figura 3, obtendo-se:

$$
\frac{P_{1}}{\gamma}=\frac{V_{3}^{2}}{2 g}+\left(Z_{3}-Z_{1}\right)+\text { HfMicrotubo }+ \text { Hf cabo }+ \text { Hf Localizada }
$$

Aplicando-se a equação de Bernoulli entre os pontos 4 e 1, tem-se:

$$
\frac{P_{1}}{\gamma}=\frac{P_{4}}{\gamma}+\left(\frac{V_{4}^{2}}{2 g}-\frac{V_{1}^{2}}{2 g}\right)+\left(Z_{4}-Z_{1}\right)-\mathrm{Hf}_{\mathrm{L}}
$$

Considerando o dimensionamento de uma linha lateral com microtubos espaçados entre si entre os ponto 4 e 1 , e um solo em desnível (dZ), em que o dimensionamento do sistema de irrigação seja realizado do último para o primeiro microtubo, o comprimento do microtubo é obtido pela eq.(16), conforme demonstrado a seguir.

Substituindo (14) em (13), e considerando as cargas de pressão $\mathrm{P} 4 / \gamma$ e $\mathrm{P} 1 / \gamma$, como sendo as pressões (P4 e P1), medidas nos pontos 4 e 1, respectivamente, tem - se:

$$
f \frac{\mathrm{L}_{2-3}}{\mathrm{D}_{2}} \frac{V_{3}^{2}}{2 g}=\left[\begin{array}{c}
\mathrm{P}_{4}-\mathrm{Hf}_{\mathrm{L}} \pm d Z-\left(Z_{3}-Z_{1}\right)+\left(\frac{V_{4}{ }^{2}}{2 g}-\frac{V_{1}{ }^{2}}{2 g}\right)- \\
\left(f \frac{\mathrm{L}_{1-2}}{\mathrm{D}_{1}} \frac{V_{2}^{2}}{2 g}+\frac{V_{3}^{2}}{2 g}+\mathrm{K} \frac{V_{3}^{2}}{2 g}\right)
\end{array}\right]
$$

Substituindo-se as eq.(10) e (11) em (15), e isolando-se o comprimento do microtubo (entre os pontos 2 e 3 ), tem-se a eq.(16): 


$$
\mathrm{L}_{2-3}=\left\{\begin{array}{c}
{\left[\mathrm{P}_{4}-\mathrm{Hf}_{\mathrm{L}} \pm \mathrm{dZ}-\left(Z_{3}-Z_{1}\right)+\left(\frac{V_{4}^{2}}{2 g}-\frac{V_{1}^{2}}{2 g}\right)\right]-} \\
{\left[\frac { 0 , 3 1 6 } { 2 \mathrm { g } } ( \frac { 4 } { \pi } ) ^ { 1 , 7 5 } \frac { v } { 1 } \left(\frac{\mathrm{Q}^{1,75} \mathrm{~L}_{1-2}}{\left.\left.\mathrm{D}_{1}^{1,75}\right)\right]-}\right.\right.} \\
{\left[\frac{16}{2 \pi^{2} \mathrm{~g}}\left(\frac{\mathrm{Q}^{2}}{\mathrm{D}_{2}^{4}}\right)\right]-\left[\frac{16}{2 \mathrm{\pi}^{2} \mathrm{~g}}\left(\mathrm{~K} \frac{\mathrm{Q}^{2}}{\mathrm{D}_{2}^{4}}\right)\right]}
\end{array}\right\}\left[\frac{1,310500}{0,316 v^{0,25}} \frac{\mathrm{gD}_{2}{ }^{4,75}}{\mathrm{Q}^{1,75}}\right]
$$

\section{Modelo 2 (WSBOTREL)}

Equação de Bernoulli aplicada entre os pontos de 1-3 e 4-1, já demonstrado, tendo-se:

$$
\begin{aligned}
& \text { HfMicrotubo }=\left[\begin{array}{c}
\left(\mathrm{P}_{4}+\frac{V_{4}^{2}}{2 g}-\frac{V_{1}^{2}}{2 g}-\mathrm{Hf}_{\mathrm{L}} \pm \mathrm{dZ}-(\mathrm{Z} 3-\mathrm{Z} 1)\right. \\
\left(\frac{V_{3}^{2}}{2 g}+\text { Hfcabo }+ \text { Hf Localizada }\right)
\end{array}\right] \\
& \mathrm{H}=\left(\mathrm{P}_{4}+\left(\frac{V_{4}^{2}}{2 g}-\frac{V_{1}^{2}}{2 g}\right)-\mathrm{HfL} \pm \mathrm{dZ}-(\mathrm{Z3}-\mathrm{Z} 1)\right) \\
& H\left(2 g D_{1} D_{2} R_{1}{ }^{0,25} R_{2}{ }^{0,25}\right)=\left[\begin{array}{c}
\left(0,316 D_{1} \mathrm{~L}_{2-3} V_{3}{ }^{2} R_{1}{ }^{0,25}\right)+ \\
\left.(K+1) V_{3}^{2} D_{1} D_{2} R_{1}{ }^{0,25} R_{2}{ }^{0,25}\right)+ \\
\left(0,316 D_{2} \mathrm{~L}_{1-2} V_{2}{ }^{2} R_{2}{ }^{0,25}\right)
\end{array}\right]
\end{aligned}
$$

Substituindo (11) e (18) em (19) e isolando-se o comprimento do microtubo, tem-se a eq.(20):

$$
\mathrm{L}_{2-3}=\left\{\left[\begin{array}{c}
{\left[\left(\mathrm{P}_{4}+\frac{V_{4}^{2}}{2 g}-\frac{V_{1}^{2}}{2 g}-\mathrm{HfL} \pm \mathrm{dZ}-(\mathrm{Z} 3-\mathrm{Z} 1)\right)\left(\frac{\mathrm{Re}_{2}{ }^{0,25} \mathrm{D}_{2}{ }^{5}}{0,026110086 \mathrm{Q}^{2}}\right)\right]-} \\
{\left[\frac{(K+1) D_{2} R_{2}{ }^{0,25}}{0,316}\right]-\left[\mathrm{L}_{1-2}\left(\frac{\mathrm{D} 2}{\mathrm{D} 1}\right)^{5}\left(\frac{\mathrm{Re}_{2}}{\mathrm{Re}_{1}}\right)^{0,25}\right]}
\end{array}\right]\right\}
$$

em que:

$\mathrm{P}_{4}$ - carga de pressão na entrada do cabo, no ponto 1, m.c.a;

$\mathrm{Hf}_{\mathrm{L}}$ - dissipação de energia na linha lateral entre os pontos 4 e 1, m.c.a;

$\mathrm{dZ}$ - diferença de nível $(\mathrm{m})$ entre os pontos 4 e $1\left(\mathrm{Z}_{4}-\mathrm{Z}_{1}\right)$;

$\mathrm{Z}_{3}$ - cota no ponto $3, \mathrm{~m}$;

$\mathrm{Z}_{1}$ - cota no ponto $1, \mathrm{~m}$;

$\mathrm{Q}$ - vazão no microtubo, $\mathrm{m}^{3} \mathrm{~s}^{-1}$;

$\mathrm{V}$ - viscosidade cinemática, $\mathrm{m}^{2} \mathrm{~s}^{-1}$;

$\mathrm{D}_{1}$ - diâmetro do cabo, m;

$\mathrm{D}_{2}$ - diâmetro do microtubo, m;

$\mathrm{L}_{1-2}$ - comprimento do cabo, $\mathrm{m}$;

$\mathrm{L}_{2-3}$ - comprimento do microtubo, $\mathrm{m}$;

$\mathrm{K}$ - constante de dissipação de energia na entrada do microtubo, adimensional;

$\mathrm{V}_{1}$ - velocidade do fluido no ponto 1 da linha lateral, $\mathrm{m} \mathrm{s}^{-1}$;

$\mathrm{V}_{4}$ - velocidade do fluido no ponto 4 da linha lateral, $\mathrm{m} \mathrm{s}^{-1}$;

$\mathrm{Re}_{1}$ - número de Reynolds no cabo, adimensional, e

$\mathrm{Re}_{2}$ - número de Reynolds no microtubo, adimensional. 
Para a realização de uma análise técnica, com auxílio dos modelos propostos, dimensionou se para sessenta diferentes pressões o comprimento dos microtubos para determinada vazão de projeto, instalados em uma linha lateral, espaçados em 0,50 m. Em seguida, coletou-se o volume de água aplicado por cada microtubo, sendo que as vazões observadas e de projeto (vazões estimadas) foram utilizadas para análise e avaliação dos modelos de acordo com as metodologias apresentadas a seguir, comparando-se os dados fornecidos pelos modelos com os dados experimentais, por meio do índice de correlação de Pearson, que representa um bom indicador de precisão, pois relaciona os dados estimados e observados (ARMINDO, 2007), índice de concordância de Willmott, erro médio absoluto e índice médio de erros, representados pelas eq.(21), (22), (23) e (24), descritas a seguir:

$$
\begin{aligned}
& r=\frac{\left(N \sum_{i=1}^{N} E i O i\right)-\left(\sum_{i=1}^{N} E i\right)\left(\sum_{i=1}^{N} O i\right)}{\sqrt{\left[\left(N \sum_{i=1}^{N}(E i)^{2}\right)-\left(\sum_{i=1}^{N} E i\right)^{2}\right]}\left[\left(N \sum_{i=1}^{N}(O i)^{2}\right)-\left(\sum_{i=1}^{N} O i\right)^{2}\right]} \\
& d=1-\frac{\sum_{i=1}^{N}(E i-O i)^{2}}{\sum_{I=1}^{N}(|E i-\bar{O}|+|O i-\bar{O}|)^{2}} \\
& E M A=\sum_{i=1}^{N} \frac{(|E i-O i|)}{N} \\
& s=\left(\frac{\sum_{i=1}^{N}(|E i-O i|)}{\sum_{i=1}^{N} E i}\right) 100
\end{aligned}
$$

em que,

$\mathrm{r}$ - coeficiente de correlação de Pearson;

$\mathrm{N}$ - número de repetições;

Ei - enésima variável estimada;

Oi - enésima variável observada;

d - índice de concordância de Willmott;

$\bar{O}$ - média das variáveis observadas;

$\mathrm{s}$ - índice médio de erro, \%, e

EMA - erro médio absoluto, $\mathrm{L} \mathrm{h}^{-1}$.

\section{RESULTADOS E DISCUSSÃO}

Os diâmetros apresentados pelas duas metodologias são mostrados na Tabela 2, com os dados de média $(\mathrm{M})$, desvio-padrão $(\sigma)$ e coeficiente de variação (C.V.).

Observando a Tabela 2, verifica-se que os valores médios de diâmetro dos microtubos V, A, L e Az, pelo método hidráulico, foram sensivelmente maiores em relação ao método do projetor de perfil. Em trabalhos realizados por SOUZA \& BOTREL (2004), o autor comenta que o diâmetro medido por meio de avaliações hidráulicas se aproxima do dos valores obtidos no projetor de perfil, sendo viável para quem não possui equipamentos especiais. Isso ocorre, principalmente, em função do comprimento do microtubo utilizado para a determinação do diâmetro, sendo que, para os testes desta pesquisa, o comprimento para avaliação hidráulica foi de até 6.000 vezes maior que o comprimento utilizado no projetor de perfil, no caso do microtubo A, sendo mais representativo. 
TABELA 2. Diâmetros dos microtubos utilizando testes hidráulicos e projetor de perfil. Diameters of microtubs using hydraulic tests and profile projector.

\begin{tabular}{|c|c|c|c|c|}
\hline \multirow{4}{*}{$\begin{array}{l}\text { Metodologia } \\
\text { de Análise }\end{array}$} & \multicolumn{4}{|c|}{ Projetor de Perfil } \\
\hline & \multicolumn{4}{|c|}{ Microtubo } \\
\hline & $\mathrm{V}$ & $\mathrm{A}$ & $\mathrm{L}$ & $\mathrm{Az}$ \\
\hline & $\mathrm{D}(\mathrm{mm})$ & $\mathrm{D}(\mathrm{mm})$ & $\mathrm{D}(\mathrm{mm})$ & $\mathrm{D}(\mathrm{mm})$ \\
\hline $\mathrm{M}$ & 0,655 & 0,708 & 0,804 & 1,015 \\
\hline $\boldsymbol{\sigma}$ & 0,023 & 0,024 & 0,021 & 0,022 \\
\hline \multirow[t]{2}{*}{ C.V. $(\%)$} & 3,471 & 3,399 & 2,625 & 2,120 \\
\hline & \multicolumn{4}{|c|}{ Testes hidráulicos } \\
\hline M & 0,702 & 0,761 & 0,893 & 1,109 \\
\hline$\sigma$ & 0,003 & 0,003 & 0,004 & 0,004 \\
\hline C.V. $(\%)$ & 0,369 & 0,427 & 0,444 & 0,335 \\
\hline
\end{tabular}

Outro fator importante é a dificuldade de leitura precisa pelo projetor de perfil, pois, uma vez sendo muito pequenos os diâmetros, por mais que se tenha cuidado, aparecem sombras na extremidade interna dos mesmos, interferindo, portanto, no resultado. Além disso, as propriedades físicas do interior do material, como altura das asperezas, influenciam na velocidade de escoamento, estando, portanto, indiretamente representada quando se trabalha com avaliação hidráulica. Portanto, para a determinação do coeficiente $\mathrm{K}$ de dissipação de energia na entrada do microtubo, utilizaram- se os diâmetros obtidos por avaliação hidráulica.

Os resultados de KV, KA, KL, e KAz para os microtubos V, A, L, Az, respectivamente, estão apresentados no gráfico em função do número de Reynolds, conforme Figura 3.

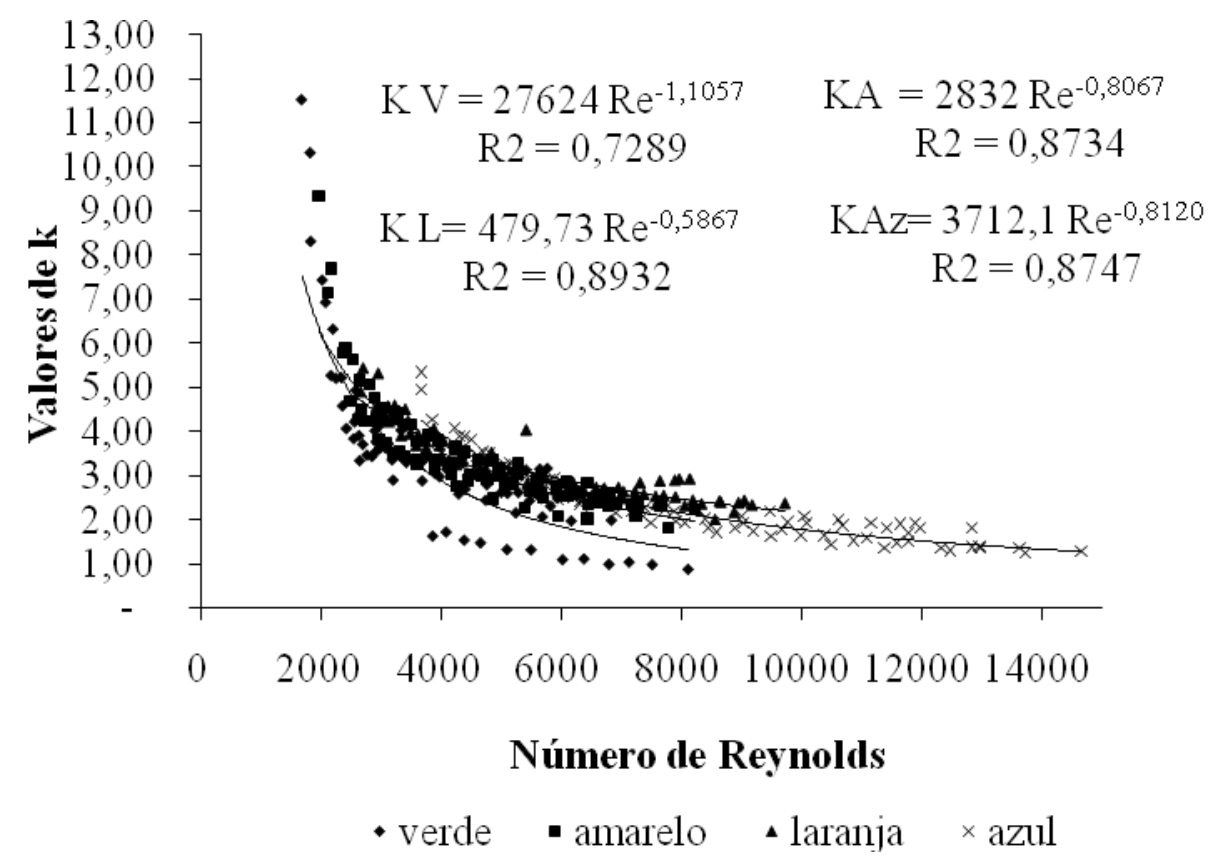

FIGURA 3. Valores de K em relação ao número de Reynolds, para os microtubos V, A, L e Az. Values of $K$ in relation to Reynolds number, for the $V, A, L$ and $A z$ microtubs.

Nota-se, pela Figura 3, que a equação potencial apresentou bom ajuste para a relação entre os valores de $\mathrm{K}$ e Reynolds, tendo, portanto, coeficientes de determinação iguais a: 0,7289 ; 0,8734; 0,8932 e 0,8747 para os microtubos V, A, L e Az, respectivamente.

Utilizando o mesmo procedimento acima para a determinação das equações que representam $\mathrm{K}$ em função de Reynolds, na Figura 4, mostram-se graficamente os dados de vazão $\left(\mathrm{L} \mathrm{h}^{-1}\right)$ 
apresentados pelos microtubos V (QV), A (QA) L (QL) e Az (QAz). Este procedimento foi realizado, tendo em vista que o número de Reynolds é um quesito para dimensionamento de microtubos sob escoamento turbulento, pelos modelos apresentados, servindo para escolha de vazão de Reynolds como dados de entrada nos modelos de dimensionamento.

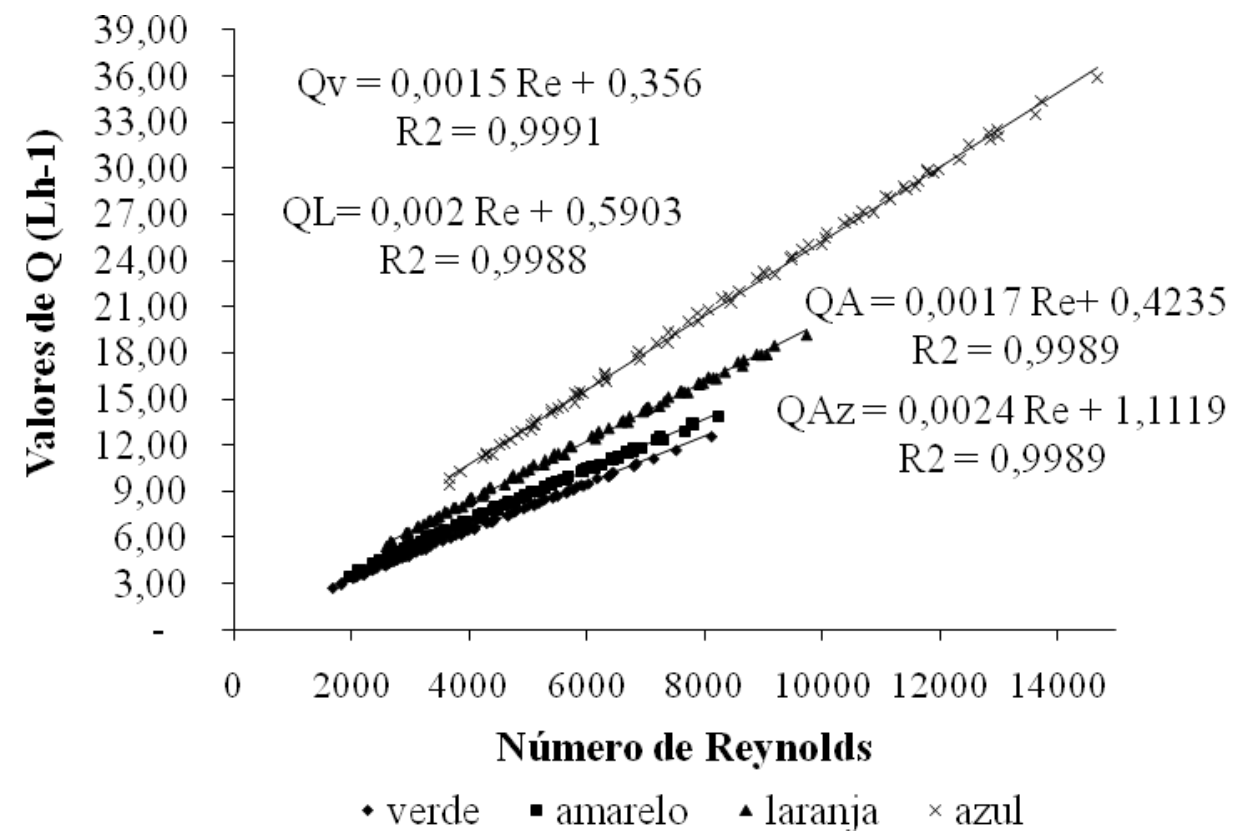

FIGURA 4. Valores de vazão em relação ao número de Reynolds para os microtubos V, A, L e Az. Values of discharge in relation to Reynolds number, for the $\mathrm{V}, \mathrm{A}, \mathrm{L}$ and $\mathrm{Az}$ microtubs.

O modelo linear descreve, com indiscutível qualidade de ajuste, os valores obtidos experimentalmente, para as condições desta pesquisa.

Nas Figuras 5 (a, b), apresentam-se graficamente os valores de vazão de projeto (estimada), utilizada no dimensionamento pelos modelos UNIVERSAL (a) e WSBOTREL (b), e observada nos testes de campo com os microtubos V, A, L e Az.
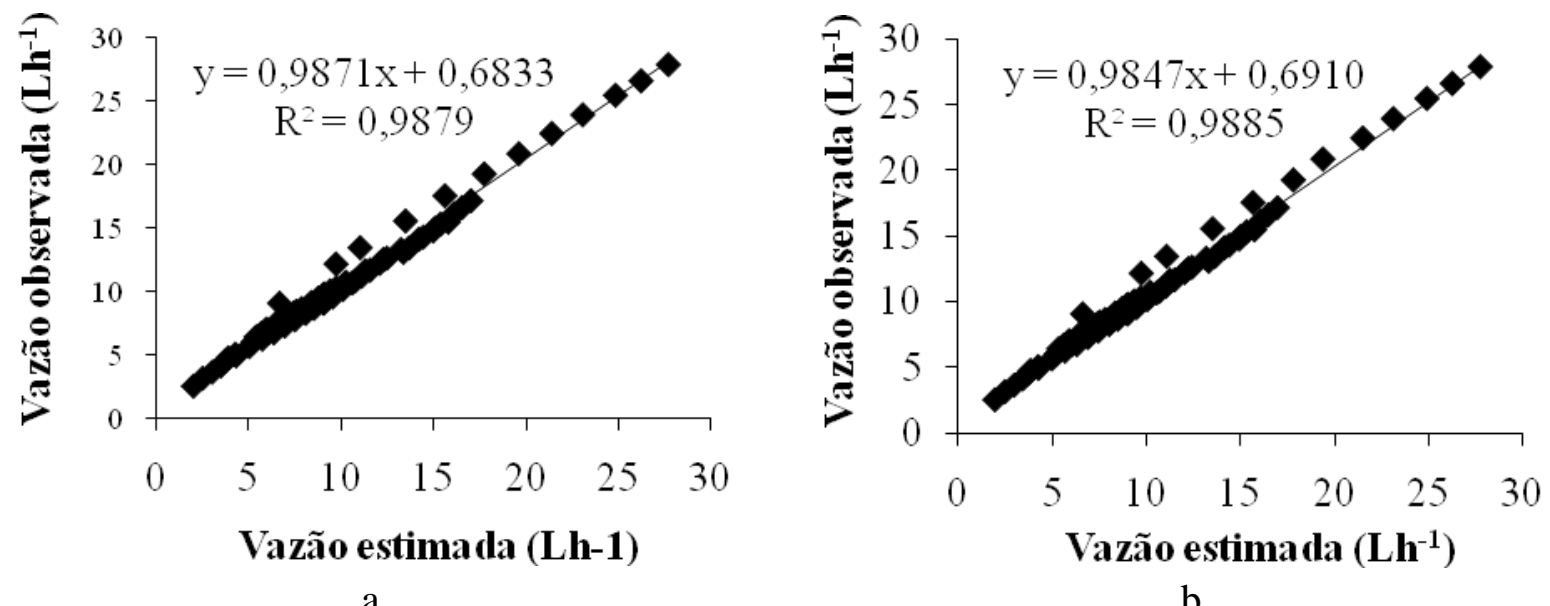

FIGURA 5. Vazão estimada e observada, utilizando os modelos UNIVERSAL (a) e WSBOTREL (b). Estimated and observed discharge, using UNIVERSAL (a) and WSBOTREL (b) models. 
A dispersão apresentada foi quase nula entre os pares de valores, tendo - se alta relação entre os dados observados e estimados, com $\mathrm{R}^{2}$ acima de $98 \%$. Apesar de termos valores altos de $\mathrm{R}^{2}$, não se garante exatidão nos dados, portanto faz-se necessária a utilização de outras análises estatísticas, conforme a seguir.

A Tabela 3 traz alguns indicadores de desempenho estatístico, sendo: coeficiente de correlação de Pearson (precisão); concordância de Willmott (exatidão); erro médio absoluto (EMA) da vazão $\left(\mathrm{Lh}^{-1}\right)$, e índice médio de erro (s) em porcentagem, calculados em função da vazão de projeto e observados em campo nos microtubos V, A, L e Az.

TABELA 3. Erro médio absoluto (EMA) da vazão $\left(\mathrm{Lh}^{-1}\right)$, correlação de Pearson, concordância de Willmott e índice médio de erros (s) em porcentagem, utilizando as vazões estimadas e observadas em campo. Mean absolute error (MAE) of discharge $\left(\mathbf{L h}^{-1}\right)$, Pearson correlation, Willmott correlation and average index of error (s) in percentage, using the estimates and observed discharges in the field.

\begin{tabular}{ccccc}
\hline \multirow{2}{*}{ Método de Análise } & \multicolumn{4}{c}{ Microtubo } \\
\cline { 2 - 4 } & $\mathrm{V}$ & $\mathrm{A}$ & $\mathrm{L}$ & $\mathrm{Az}$ \\
\hline & 0,9934 & 0,9702 & 0,9971 & 0,9898 \\
Pearson & 0,9820 & 0,9791 & 0,9931 & 0,9849 \\
Willmot & 0,4939 & 0,5943 & 0,3279 & 0,9222 \\
EMA & 7,5430 & 7,9420 & 3,1101 & 5,4600 \\
s & \multicolumn{4}{c}{ Modelo WSBOTREL } \\
\hline Pearson & 0,9928 & 0,9679 & 0,9975 & 0,9853 \\
Willmot & 0,9827 & 0,9792 & 0,9938 & 0,9860 \\
EMA & 0,4749 & 0,5693 & 0,3129 & 0,8812 \\
s & 7,2320 & 7,5830 & 2,9603 & 5,2000 \\
\hline
\end{tabular}

De acordo com os valores apresentados na Tabela 3, por meio de ambos os modelos, obtevese ótimo desempenho. Os valores de vazão foram estimados precisa e exatamente acima de $96 \%$ e 97\%, quando se utilizaram o coeficiente de correlação de Pearson e o índice de concordância de Willmott, respectivamente, para todos os microtubos.

O erro médio absoluto também teve valores parecidos entre os modelos, sendo que o modelo WSBOTREL apresentou resultados ligeiramente inferiores ao UNIVERSAL. O maior EMA ocorreu quando se utilizou o microtubo Az, que apresenta maior diâmetro, entretanto não se pode dizer que este erro aumenta em função de se ter maior diâmetro, pois existem outras variáveis interferentes, como é o caso da variação na medida do diâmetro do microtubo, valores de Reynolds e vazão elevados, tornando-se sensível a variação de vazão, principalmente no microtubo Az que tem maior diâmetro . Além disso, os valores do coeficiente $\mathrm{K}$ de dissipação de energia localizada, inseridos na equação que determina os comprimentos dos microtubos, foram estimados por uma equação, conforme mostra a Figura 3; isso permite, também, uma pequena variação em função desta variável, tendo em vista que os coeficientes de determinação das equações foram menores que um. Analisando o índice médio de erros, nota-se que os valores apresentados quando se utilizou o modelo WSBOTREL, foram sensivelmente menores para todos os microtubos, quase não sendo notada diferença. Por outro lado, a maior variação no índice médio de erros foi de 7,94\% para o microtubo A, utilizando o modelo UNIVERSAL, o que significa excelência em termos de campo.

A pequena diferença de erros entre os modelos pode ser explicada pelo fato de que, quando se trabalha com um modelo gerado a partir de testes de laboratório, aumenta-se a chance de utilizar, de maneira mais correta, variáveis como rugosidade relativa do material, estando a mesma representada indiretamente no modelo WSBOTREL. Entretanto, para o modelo UNIVERSAL, esta variável não é expressa como dado de entrada na equação. 


\section{CONCLUSÕES}

Dois modelos matemáticos para dimensionamento de microtubos em regime de escoamento turbulento foram desenvolvidos (UNIVERSAL e WSBOTREL), obtendo-se excelente desempenho, podendo ambos ser utilizados.

Os coeficientes de dissipação de energia localizada estimados podem ser utilizados para dimensionamento de microtubo em condições de regime de escoamento turbulento, e o diâmetro deverá ser aquele determinado por avaliações hidráulicas.

A avaliação do sistema de irrigação com microtubos operando em regime de escoamento turbulento, em uma linha lateral em campo, mostrou que a utilização dos modelos para dimensionamento dos comprimentos de todos os modelos de microtubos estudados, utilizando o coeficiente $\mathrm{K}$ de dissipação de energia localizada, foi precisa e exata a $96 \%$ e $97 \%$, quando se utilizaram o coeficiente de correlação de Pearson e o índice de concordância de Willmott, respectivamente.

\section{AGRADECIMENTOS}

À ESALQ/USP, pela estrutura e área experimental; ao professor Tarlei Arriel Botrel, pela orientação, e aos amigos da pós-graduação. À FAPESP, pelo apoio financeiro.

\section{REFERÊNCIAS}

AIROLDI, R.P. da S. Eficiência dos meios filtrantes disco e manta sintética não tecida em fertirrigação por gotejamento. 2003. 204 f. Dissertação (Mestrado em Água e Solo) - Universidade de Campinas, Campinas, 2003.

ARMINDO, R.A. Avaliação do desempenho e da durabilidade dos componentes do sistema de pulverização Notliada. 2007. 70 f. Dissertação (Mestrado em Irrigação e Drenagem) - Escola Superior de Agricultura “Luiz de Queiroz”, Universidade de São Paulo, Piracicaba, 2007.

CARARO, D.C.; BOTREL, T.A. Uso de cloração e ar comprimido no controle de entupimento de gotejadores ocasionado pela aplicação de água residuária. Engenharia Agrícola, Jaboticabal, v.27, n.2, p.336-345, 2007.

CHRISTOFIDIS, D. Agricultura irrigada sustentável no Semiárido e no Rio Grande do Norte. Irrigação e Tecnologia Moderna, Brasília, v.1, n.74/75, p.62-67, 2007.

NASCIMENTO, T. Caracterização Hidráulica do Microaspersor Rain-Bird Qn-141. Revista Brasileira de Engenharia Agrícola e Ambiental, Campina Grande, v.3, n.1, p.30-33, 1999.

SAAD, J.C.C.; MARCUSSI, F.F.N. Distribuição da carga hidráulica em linhas de derivação otimizadas por programação linear. Engenharia Agrícola, Jaboticalbal, v.26, n.2, p.406-414, 2006.

SOUZA, I.H. de; ANDRADE,E.M.de; SILVA, E.L.da. Avaliação hidráulica de um sistema de irrigação localizada de baixa pressão, projetado pelo software "bubbler". Engenharia Agrícola. Jaboticabal, v.25, n.1, p.264-271, 2005.

SOUZA, R.O.R.M.; BOTREL, T.A. Modelagem para dimensionamento de microtubo em irrigação localizada. Revista Brasileira de Engenharia Agricola e Ambiental, Campina Grande, v.8,n.1, p.16$22,2004$.

VERMEIREN, L.; JOBLING, G.A. Localized Irrigation. Design, Installation, Operation, Evaluation. Irrigation and Drainage, Roma, n.36, p.203, 1980.

ZANETTI, S.S.; PRUSKI, F.F.; MOREIRA, M.C.; DEDIYAMA, G.C. ; SILVA, D.D. Programa computacional para geração de séries sintéticas de precipitação. Engenharia Agrícola, Jaboticabal, v.25, n.1,p.96-104, 2005. 